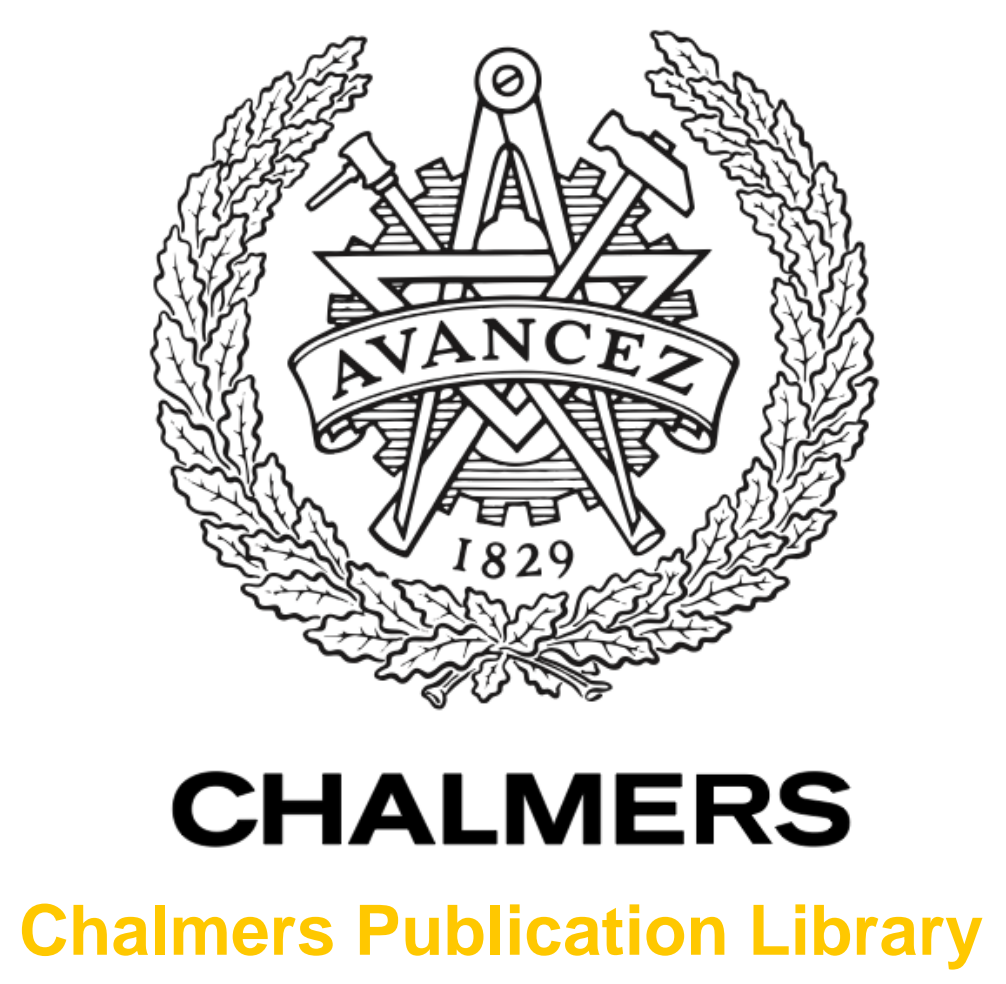

\title{
Resonance Based Target Recognition Using Ultra-Wideband Polarimetric Signatures
}

This document has been downloaded from Chalmers Publication Library (CPL). It is the author's version of a work that was accepted for publication in:

IEEE Transactions on Antennas and Propagation (ISSN: 1045-9243)

Citation for the published paper:

Lui, H. ; Shuley, N. (2012) "Resonance Based Target Recognition Using Ultra-Wideband Polarimetric Signatures". IEEE Transactions on Antennas and Propagation, vol. 60(8), pp. 3985-3988.

http://dx.doi.org/10.1109/TAP.2012.2201074

Downloaded from: http://publications.lib.chalmers.se/publication/157340

Notice: Changes introduced as a result of publishing processes such as copy-editing and formatting may not be reflected in this document. For a definitive version of this work, please refer to the published source. Please note that access to the published version might require a subscription. 


\section{Resonance Based Target Recognition Using Ultrawideband Polarimetric Signatures}

Hoi-Shun Lui and Nicholas V. Shuley

\begin{abstract}
Automated target recognition based on resonances embedded in the ultrawideband transient signatures has been of significant research interest throughout the years. In most studies, targets are usually illuminated and measured using a linear polarized basis. This could lead to an incorrect result for target recognition as some resonant modes may not be well excited at that particular incident aspect and polarization state. In this communication, the possibility of using a full-polarimetric ultrawideband target signature is investigated. Target recognition of some simple wire targets using the extinction pulse technique demonstrate that it would be easier to fully excite all of the important resonances using a circular polarization basis with due consideration of the co- and cross-polarized target signatures.
\end{abstract}

Index Terms-Polarization, resonance-based target recognition, transient electromagnetic scattering, ultrawideband radar.

\section{INTRODUCTION}

Over the years target recognition based on the natural resonant frequencies (NRFs) embedded in transient electromagnetic target responses has been of significant interest. These NRFs are purely dependent on the physical properties of the target and are independent of both incident aspect and polarization state [1], [2]. The aspect and polarization independence nature allows these NRFs to be an excellent candidate for target classification. Automated target recognition (ATR) algorithms such as E-Pulse [3]-[6] were introduced and applied to targets in free space. Recent attempts have been focused on ATR algorithms based on partial/substructure resonances [7]-[9].

To our knowledge, most studies consider the case of an ultrawideband (UWB) transient signature obtained under a single linear polarization excitation and the corresponding co-polarized scattered field is measured. Recent work by Shuley et al. [2] considered the polarization dependency of the NRFs. The results showed that the residues of the extracted NRFs vary as a function of the incident linear polarization angle. At some polarization angles, the residues of the NRFs could be so small such that the NRFs are not found in the extraction process. In an ATR scenario, the target orientations are not usually known a priori and the chances of the entire dominant NRFs being strongly excited become uncertain. This is one key limitation and weakness of resonance based target recognition. One way to handle this issue is to illuminate the target from a number of linear polarization angles. Especially in ATR of similar targets, it is important that the partial/substructure resonances are well excited [7]. Lui and Shuley [10] investigated different ways of extracting and processing the NRFs from a number of transient signatures obtained from different linear polarization angles. It was concluded that the most accurate approach was probably to obtain

Manuscript received March 01, 2011; revised December 28, 2011; accepted February 25, 2012. Date of publication May 24, 2012; date of current version July 31, 2012.

H.-S. Lui is with the Department of Signals and Systems, Chalmers University of Technology, SE412 96, Gothenburg, Sweden (e-mail: antony.lui@chalmers.se; lui@ieee.org).

N. V. Shuley is with the School of Information Technology and Electrical Engineering, The University of Queensland, St. Lucia, Queensland 4072, Australia (e-mail: shuley@itee.uq.edu.au).

Color versions of one or more of the figures in this communication are available online at http://ieeexplore.ieee.org.

Digital Object Identifier 10.1109/TAP.2012.2201074 a set of NRFs based on data from all linear polarized incident excitations. [11]. However, the price paid is a relatively high computational cost as the number of target signature increases in order to reliably extract all the dominant NRFs.

In this communication, the prospect of using different UWB polarization responses for target recognition is investigated. Without the loss of generality, linear and circular polarized UWB target signatures are considered. Numerical examples of some simple targets will be used to demonstrate the advantages of using polarimetric target signatures. The results show that the residues of the NRFs become less orientation dependent and the dominant NRFs can be extracted from only one polarimetric target signature (low computational cost) without the risk of data corruption and omission of NRF in the selection process [10].

\section{RESONANCE BASED TARgEt RECOGNITION}

Upon excitation of the target in free space using a short electromagnetic pulse, the late time of the target signature can be expressed as [1]

$$
r(t)=\sum_{n=1}^{N} a_{n} e^{\sigma_{n} t} \cos \left(\omega_{n} t+\phi_{n}\right), \quad t>T_{l}
$$

where $a_{n}$ and $\varnothing_{n}$ are the aspect dependent residues and phase of the $n^{\text {th }}$ mode respectively and $T_{l}$ is the onset of the late time period. It is assumed that only $N$ modes are excited under band-limited short-pulse excitation. The NRFs are given by $s_{n}=\sigma_{n} \pm j \omega_{n}$, where $\sigma_{n}$ and $\omega_{n}$ are the damping coefficients and resonant frequencies respectively.

If we describe the entire scattering problem at a particular frequency numerically via an integral equation formulation, it is well known that the integral equation can be written in matrix form and solved via moment method. In general, it can be written as

$$
[\mathbf{Z}][\mathbf{I}]=[\mathbf{V}]
$$

where $[\mathbf{Z}]$ is the impedance matrix that corresponds to the target geometry, $[\mathbf{I}]$ is a column vector corresponding to the unknown current induced on the target and $[\mathbf{V}]$ is a column vector corresponds to the excitation. According to the singularity expansion method (SEM), the NRFs correspond to the singularities of the $[\mathbf{Z}]$ matrix and can be extracted using a typical (complex) root searching method such as Muller's method [1]. It is apparent that the NRFs are purely dependent on the target geometry and they are independent of the excitation vector $[\mathbf{V}]$. However, the residues of the NRFs do vary as a function of the excitation vector. This explains the situation why some NRFs cannot be retrieved at certain transmitting/receiving configurations [12].

\section{NUMERICAL EXAMPLES}

To investigate how the excitation and receiving polarization states affect the performance of resonance based target recognition, numerical examples of three wire targets were studied. These consisted of a vertical wire segment (main body) of $1 \mathrm{~m}$ and a horizontal wire segment of $r=0.3 \mathrm{~m}$ (Target 1) and $0.2 \mathrm{~m}$ (Target 2) respectively, located at the centre of the main body, as shown in Fig. 1(a). Target 3 has the same horizontal wire segment of $0.3 \mathrm{~m}$ except it is located at $0.2 \mathrm{~m}$ away from the end of the main body, as shown in Fig. 1(b). The targets are excited with linear polarized plane wave with vertical (V) and horizontal $(\mathrm{H})$ polarization. The electromagnetic scattering problems are solved using moment method solver FEKO [13] in the frequency domain from $3.9 \mathrm{MHz}$ to $2 \mathrm{GHz}$ with 512 equally spaced samples. The thin wire approximation is used here with the radius of 10 


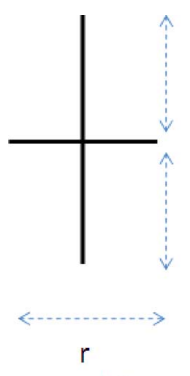

(a)

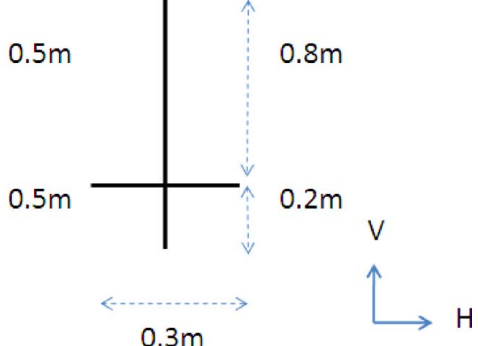

(b)
Fig. 1. Wire targets and corresponding excitation polarization references. (a) Target 1: $r=0.3 \mathrm{~m}$, Target 2: $r=0.2 \mathrm{~m}$ and (b) Target 3 .

$\mu \mathrm{m}$. The scattered far-fields of both co- and cross-polarization are measured in the monostatic direction. The four components in the Sinclair scattering matrix [15], $[S(H, V)]=\left[\begin{array}{ll}S_{H H} & S_{H V} \\ S_{V H} & S_{V V}\end{array}\right]$, that relates the scattered far field with an incident plane wave with linear polarization in the frequency domain, are obtained. Using the transformation described in [15], the scattering matrices in the circular polarization basis $[S(L, R)]=\left[\begin{array}{ll}S_{L L} & S_{L R} \\ S_{R L} & S_{R R}\end{array}\right]$ at each frequency sample can be determined. Here, $L$ and $R$ correspond to left-hand and right-hand circular polarization respectively. To obtain the corresponding scattering matrices in time domain, i.e., $\left[S_{(H, V)}(t)\right]=\left[\begin{array}{ll}S_{H H}(t) & S_{H V}(t) \\ S_{V H}(t) & S_{V V}(t)\end{array}\right]$ and $\left[S_{(L, R)}(t)\right]=\left[\begin{array}{ll}S_{L L}(t) & S_{L R}(t) \\ S_{R L}(t) & S_{R R}(t)\end{array}\right]$, the frequency samples are first windowed via a Gaussian window [14] and inverse Fourier transformed to the time domain. Under the monostatic configuration, $S_{L L}(t)=$ $S_{R R}(t)$ and $S_{L R}(t)=S_{R L}(t)$ and thus we only need to consider $S_{L L}(t)$ and $S_{L R}(t)$. Here, the wire segments are perpendicular to each other. Under vertical excitation, the main body is excited but not the horizontal wire segment. Theoretically, the cross-polarized response should be zero in this case and vice versa for horizontal polarization excitation and thus the $S_{H V}(t)$ and $S_{V H}(t)$ responses are not considered.

\section{A. NRF of the Wire Targets}

The fundamental resonant frequency for a simple wire segment with length $l$ can be approximated by $f_{R}=c /(2 \times l)$, where $c=3 \times$ $10^{8} \mathrm{~m} / \mathrm{s}$ is the speed of light in free-space. The resonant frequencies of different wire lengths that correspond to the targets are listed in Table I, which gives us some idea of what the extracted NRFs should be and what are they physically corresponding to. To extract the NRF from the polarimetric transient signatures, late time samples from $12 \mathrm{~ns}$ to $137 \mathrm{~ns}$ are imported to the matrix pencil method [16]. To quantify the energy level of each NRF with respect to the overall energy of the target signature, the Energy Ratio $\left(E R_{n}\right)$ is used and it is defined as

$$
E R_{n}=\frac{\int_{t=T_{L}}^{T_{L}+W}\left|a_{n} e^{\left(\sigma_{n}+j \omega_{n}\right) t}\right|^{2}}{\sum_{n=1}^{N}\left[\int_{t=T_{L}}^{T_{L}+W}\left|a_{n} e^{\left(\sigma_{n}+j \omega_{n}\right) t}\right|^{2}\right]} \times 100 \%
$$

where the numerator corresponds to the energy level of a particular resonant mode $n$ and the denominator corresponds to the sum of the energy level of all the resonant modes. The extracted NRFs from $S_{V V}(t)$ and $S_{H H}(t)$ are listed in Table II. The NRFs that correspond to the 1 $\mathrm{m}$ wire and horizontal wire segments as well as the spurious modes are denoted as $*$, \# and ${ }^{\wedge}$ respectively. Compared with the estimated resonant frequencies in Table I, it can be seen that mode 1, 2 and 4 corre-
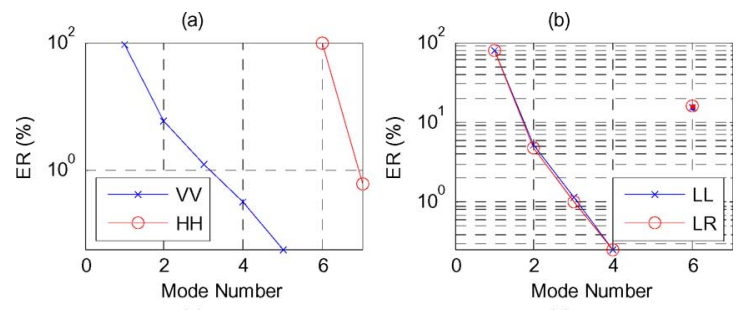

(c)
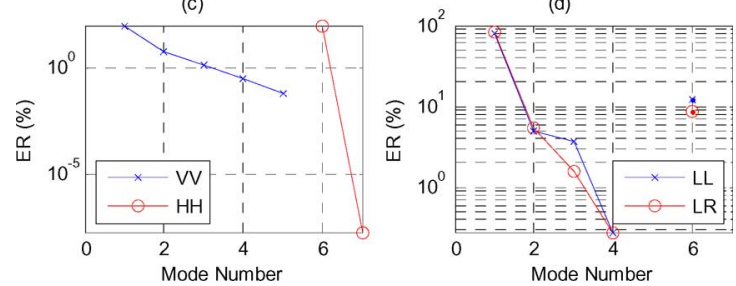

Fig. 2. $E R_{n}$ for the NRFs of the targets under different polarization states. (a) Target 1. (b) Target 1. (c) Target 2. (d) Target 2.

TABLE I

LENGTHS OF WiRe SEgMENTS AND THEIR CORRESPONDING HALF WAVELENGTH RESONANT FREQUENCIES

\begin{tabular}{|c|c|c|c|}
\hline$l=\frac{\lambda}{2}$ & $f_{R}$ & $\frac{\omega_{n}}{c}=\frac{2 \pi f_{R}}{c}$ & Corresponds to \\
\hline $1 \mathrm{~m}$ & $150 \mathrm{MHz}$ & 3.14 & Main body \\
\hline $0.33 \mathrm{~m}$ & $455 \mathrm{MHz}$ & 9.52 & Main body \\
\hline $0.3 \mathrm{~m}$ & $500 \mathrm{MHz}$ & 10.47 & Target 1,3 \\
\hline $0.2 \mathrm{~m}$ & $750 \mathrm{MHz}$ & 15.70 & Target 2 \\
\hline $0.15 \mathrm{~m}$ & $1 \mathrm{GHz}$ & 20.94 & Main body \\
\hline $0.1 \mathrm{~m}$ & $1.5 \mathrm{GHz}$ & 31.42 & Target 1,3 \\
\hline
\end{tabular}

spond to the fundamental and higher order resonances of the $1 \mathrm{~m}$ main body of the three targets and mode 6 corresponds to the fundamental resonance of the horizontal wire segment of each target. As listed in Table II, it is found that modes 1 to 5 are extracted from $S_{V V}(t)$ for Target 1 and 2 and they are identical. The corresponding $E R_{n}$ values are shown in Fig. 2(a) and (c) and found that most of the energy corresponds to mode 1 . For the case of $S_{H H}(t)$, only modes 6 and 7 are excited with almost all the energy contributed to mode 6 , which is the fundamental mode for the horizontal wire segment. Modes 5 and 7 have less than $1 \%$ of $E R_{n}$ and they are not found in other polarization states. They are spurious modes resulting from the extraction process.

The corresponding results under circular polarization basis are shown in Table II and Fig. 2. Modes 1 to 4, which correspond to the NRF of the $1 \mathrm{~m}$ main body, are well excited in both $S_{L L}(t)$ and $S_{L R}(t)$ for Target 1 and 2 with more than $80 \%$ of energy for the targets. Mode 6 for Target 1 and 2 (marked as \#), corresponding to the short wire segment of each target, are reasonably well illuminated with more than $15 \%$ and $8 \%$ of energy respectively. To sum up, when the targets are illuminated using linear polarization, some NRFs could be weakly excited. It can be seen visually in Fig. 2(a) and (c) that only mode 1 to 4 are excited for $S_{V V}(t)$ and mode 6 for $S_{H H}(t)$. When the target is illuminated using circular polarization, it is clearly shown in Table II and Fig. 2(b) and (d) that the NRF for both the $1 \mathrm{~m}$ main body and the horizontal wire segment, i.e., modes 1 to 4 and 6 , are well illuminated.

\section{B. Target Recognition via E-Pulse}

To probe further, the E-Pulse technique and the target signatures under different polarization basis are used to investigate the ATR performance under different polarization states. The E-Pulses for each 
TABLE III

ATR Using Target Signatures Under Different Polarization Basis Using E-Pulse Technique With THE CORRESPONDING $D R_{p, q}(\mathrm{DB}) \operatorname{VALUES}(p \neq q)$

\begin{tabular}{|c|c|c|c|c|c|c|c|c|c|c|c|c|}
\hline \multirow{2}{*}{$\begin{array}{c}D R_{p, q} \\
(\mathrm{~dB})\end{array}$} & \multicolumn{3}{|c|}{ (a) $S_{V V}(t)$} & \multicolumn{3}{c|}{ (b) $S_{H H}(t)$} & \multicolumn{3}{c|}{ (c) $S_{L L}(t)$} & \multicolumn{3}{c|}{ (d) $S_{L R}(t)$} \\
\cline { 2 - 43 } & $p=1$ & $p=2$ & $p=3$ & $p=1$ & $p=2$ & $p=3$ & $p=1$ & $p=2$ & $p=3$ & $p=1$ & $p=2$ & $p=3$ \\
\hline$q=1$ & 0 & 0.01 & 42.9 & 0 & 46.6 & 0.002 & 0 & 31.4 & 36.0 & 0 & 38.9 & 42.9 \\
\hline$q=2$ & -0.01 & 0 & 42.9 & 126.2 & 0 & 126.2 & 27.0 & 0 & 43.8 & 26.7 & 0 & 44.6 \\
\hline$q=3$ & 65.4 & 65.4 & 0 & -0.002 & 53.9 & 0 & 48.4 & 53.1 & 0 & 43.6 & 49.3 & 0 \\
\hline
\end{tabular}

TABLE II

EXTRACTED NRFS OF TARGET 1 AND 2

\begin{tabular}{|c|c|c|}
\hline$n$ & Target 1 & Target 2 \\
\hline 1 & $-0.11 \pm j 3.06(\mathrm{VV}, \mathrm{LL}, \mathrm{LR})^{*}$ \\
\hline 2 & \multicolumn{2}{|c|}{$-0.17 \pm j 9.32(\mathrm{VV}, \mathrm{LL}, \mathrm{LR})^{*}$} \\
\hline \multirow{2}{*}{3} & $-0.21 \pm j 15.61(\mathrm{VV})^{*}$ \\
\cline { 2 - 3 } & $-0.21 \pm j 15.61(\mathrm{LL})^{*}$ & $-0.24 \pm j 15.60(\mathrm{LL})^{*}$ \\
\cline { 2 - 3 } & $-0.23 \pm j 15.61(\mathrm{LR})^{*}$ & $-0.47 \pm j 15.81(\mathrm{LR})^{*}$ \\
\hline 4 & $-0.24 \pm j 21.93(\mathrm{VV}, \mathrm{LL}, \mathrm{RR})^{*}$ \\
\hline 5 & $-0.22 \pm j 28.31(\mathrm{VV})^{\wedge}$ \\
\hline 6 & $-0.40 \pm j 10.18(\mathrm{HH}) \#$ & $-0.63 \pm j 15.26(\mathrm{HH}) \#$ \\
& $-0.40 \pm j 10.18(\mathrm{LL}) \#$ & $-0.62 \pm j 15.30(\mathrm{LL}) \#$ \\
& $-0.39 \pm j 10.17(\mathrm{LR}) \#$ & $-0.80 \pm j 15.51(\mathrm{LR}) \#$ \\
\hline 7 & $-0.64 \pm j 31.17(\mathrm{HH})^{\wedge}$ & $-0.02 \pm j 15.02(\mathrm{HH})^{\wedge}$ \\
\hline
\end{tabular}

target at each polarization state are constructed using the extracted NRF at each polarization state [4] and then convolved with the target signatures from different targets but with the same polarization state. Both the E-Pulses and target signatures are re-sampled as usually the sampling rate of the E-Pulse and the target signatures are not the same [6]. Here, the Discrimination Ratio $\left(D R_{p, q}\right)$ is used to quantify the ATR performance, given by [3], [6], [7]

$$
D R_{p, q}=10 \log _{10}\left[\frac{\left[\int_{T_{L}}^{T_{L}+W}\left(e_{q}(t) * r_{p}(t)\right)^{2} d t\right]}{\left[\int_{0}^{T_{e}} e_{q}^{2}(t) d t\right]}\right]
$$

where $W$ is the duration of the observation window. The results are tabulated in Table III. With almost identical NRFs extracted from the target signatures, the E-Pulse technique fails to recognize between Target 1 and 2 for the case of $S_{V V}(t)$, with $D R_{1,2}$ and $D R_{2,1}$ values near to $0 \mathrm{~dB}$. For the case of $S_{H H}(t)$, the horizontal wire segments of the two targets are well excited and $D R_{1,2}$ and $D R_{2,1}$ values of $46.6 \mathrm{~dB}$ and $126.2 \mathrm{~dB}$ are obtained, which indicates successful target recognition. For the case of Target 1 and 3, both have the same horizontal wire segment and the E-Pulse technique failed to discriminate the two targets. For the case of $S_{V V}(t)$, the different positions of the horizontal wire segments resulted in different current distributions on their main bodies. The E-Pulses successfully distinguish between Target 1 and 3 with positive $D R_{p, q}$ values of $42.9 \mathrm{~dB}$ and $65.4 \mathrm{~dB}$. Comparing Target 2 and 3 with different positions and length of the horizontal wire segments, the E-Pulse technique is able to distinguish between the two targets for both $S_{V V}(t)$ and $S_{H H}(t)$.

When the NRFs of both the targets' main body and substructure are well excited under circular polarization, positive values of $D R_{p, q} \mathrm{~s}$ result for all cases with values of at least $26 \mathrm{~dB}$, which indicates the E-Pulse technique can successfully discriminate the three targets. Such results indicate that using UWB target signatures with circular polarization states can result in a more stable target recognition solution with positive values of $D R_{p, q}$ resulting for all cases.

\section{CONCLUSIONS}

The possibility of using different polarization states in the UWB ATR has been investigated. Numerical examples of three wire targets under linear and circular polarization states were considered. It was found that the use of circular polarization states can effectively excite the dominant NRFs that corresponds both the main body and the substructure of the targets regardless of the polarization angle in linear polarization states. This leads to more reliable results for target classification and ATR using E-Pulse. Compared to previous studies that require a large number of target signatures (more than 10 [2]) using different polarization angles, only four UWB polarimetric signatures (three for monostatic configuration) are required and NRF extraction is performed only on one target signature, which partially removes the issues of data corruption, NRF selection and computational load. The example presented here deals with targets that have strong linear polarimetric responses. For complicated targets that have strong linear and circular polarimetric response, the use of one polarimetric response may not be sufficient. An alternative solution is to extract the NRFs based on the four polarimetric signatures (or three for monostatic configuration) using the modified MPM algorithm [11]. Computationally this is still less than the case of using more than 10 linear polarized signatures in the extraction process. In summary, this work has opened up another possibility of exploiting full polarimetric data in the context of UWB resonance based target recognition.

\section{REFERENCES}

[1] C. E. Baum, "The singularity expansion method," in Transient Electromagnetic Fields, L. B. Felsen, Ed. New York: Springer-Verlag, 1976, pp. $129-179$.

[2] N. Shuley and I. D. Longstaff, "Role of polarisation in automatic target recognition using resonance descriptions," Electron. Lett., vol. 40, no. 4, pp. 268-270, Feb. 2004.

[3] P. Ilavarasan, J. E. Ross, E. J. Rothwell, K. M. Chen, and D. P. Nyquist, "Performance of an automated radar target pulse discrimination scheme using E pulses and S pulses," IEEE Trans. Antennas Propag., vol. 41, no. 5, pp. 582-588, May 1993.

[4] E. Rothwell, K. M. Chen, D. P. Nyquist, and W. Sun, "Frequency domain E-pulse synthesis and target discrimination," IEEE Trans. Antennas Propag., vol. 35, no. 4, pp. 426-434, Apr. 1987.

[5] A. Gallego, M. C. Carrion, D. P. Ruiz, and A. Medouri, "Extended E-pulse for discrimination of conducting spheres," IEEE Trans. Antennas Propag., vol. 41, no. 10, pp. 1460-1462, Oct. 1993.

[6] H. S. Lui and N. V. Z. Shuley, "Sampling procedures in resonance based radar target identification," IEEE Trans. Antennas Propag., vol. 56, no. 5, pp. 1487-1491, May 2008.

[7] H. S. Lui and N. V. Z. Shuley, "Radar target identification using a banded E-pulse technique," IEEE Trans. Antennas Propag., vol. 54, no. 12 , pp. $3874-3881$, Dec. 2006.

[8] C. E. Baum, "Substructure SEM," in Ultra-Wideband Short-Pulse Electromagnetics, F. Sabath, Ed. et al. New York: Springer, 2007, vol. 7, ch. 72, pp. 681-689. 
[9] H. S. Lui and N. V. Z. Shuley, "Evolutions of partial and global resonances in transient electromagnetic scattering," IEEE Antennas Wireless Propag. Lett., vol. 7, pp. 435-439, 2008.

[10] H. S. Lui and N. Shuley, "Resonance based radar target identification with multiple polarizations," in Proc. IEEE Antennas Propag. Society Int. Symp., Albuquerque, NM, Jul. 9-14, 2006, pp. 3259-3262.

[11] T. K. Sarkar, S. Park, J. Koh, and S. M. Rao, "Application of the matrix pencil method for estimating the SEM (singularity expansion method ) poles of source-free transient responses from multiple look directions," IEEE Trans. Antennas Propag., vol. 48, no. 4, pp. 612-618, Apr. 2000.

[12] H. S. Lui and N. Shuley, "On the analysis of electromagnetic transients from radar targets using smooth pseudo Wigner-Ville distribution (SPWVD)," in Proc. IEEE Antenna Propag. Society Int. Symp., Honolulu, HI, Jun. 10-15, 2007, pp. 5701-5704.

[13] FEKO EM Software \& Systems S.A., Ltd. Technopark, Stellenbosch, South Africa.

[14] S. M. Rao, Time Domain Electromagnetics. San Diego: Academic Press, 1999.

[15] A. P. Agrawal and W. M. Boerner, "Redevelopment of Kennaugh's target characteristic polarization state theory using the polarization transformation ratio formalism for the coherent case," IEEE Trans. Geosci. Remote Sensing, vol. 27, no. 1, pp. 2-14, Jan. 1989.

[16] T. K. Sarkar and O. Pereira, "Using the matrix pencil method to estimate the parameters of a sum of complex exponentials," IEEE Antennas Propag. Mag., vol. 37, no. 1, pp. 48-55, Feb. 1995. 